\title{
Geosciencences
}

http://dx.doi.org/10.1590/0370-44672015690139

Edgar Batista Medeiros Júnior

Professor Assistente

Universidade Federal do Espírito Santo - UFES

Departamento de Geologia

Alegre - Espírito Santo - Brasil

edgarjr@ymail.com

\section{Reik Degler}

Doutorando da Universidade Federal de Minas

Gerais - Departamento de Geociências -

Programa de Pós-Graduação em Geologia

Belo Horizonte - Minas Gerais - Brasil

reikdegler@gmail.com

\section{Hanna Jordt-Evangelista}

Professora Titular

Universidade Federal de Ouro Preto - UFOP

Escola de Minas, Departamento de Geologia

Ouro Preto - Minas Gerais - Brasil

hanna@degeo.ufop.br

\section{Gláucia Nascimento Queiroga}

Professora Adjunta

Universidade Federal de Ouro Preto - UFOP

Escola de Minas, Departamento de Geologia

Ouro Preto - Minas Gerais - Brasil

glauciaqueiroga@yahoo.com.br

\section{Bernhard Schulz}

\section{Professor}

TU Bergakademie - Institute of Mineralogy

Freiberg - Saxony - Germany

bernhard.schulz@mineral.tu-freiberg.de

\section{Rodson Abreu Marques}

Professor Adjunto

Universidade Federal do Espírito Santo - UFES

Departamento de Geologia

Alegre - Espírito Santo - Brasil

rodson.marques@ufes.br

\section{Introduction}

The Acaiaca Complex (AC) is located near the town of Acaiaca in the central-southeastern region of Minas Gerais state (Figure 1). The granulites of the AC are distributed along a narrow strip within the amphibolite facies gneisses of the Mantiqueira Complex.

\section{Electron microprobe Th-U-Pb monazite dating and metamorphic evolution of the Acaiaca Granulite Complex, Minas Gerais, Brazil}

\begin{abstract}
The Acaiaca Complex (AC) is located in southeastern Minas Gerais state, and comprises felsic, mafic, ultramafic, and aluminous granulites as well as lower grade gneisses and mylonites. The complex is distributed over an area of ca. $36 \mathrm{~km}$ by $6 \mathrm{~km}$, surrounded by amphibolite facies gneisses of the Mantiqueira Complex (MC). The discrepancy in the metamorphic grade between both complexes led to the present study aiming to understand the metamorphic history of the AC by means of geothermobarometric calculations and electron microprobe $\mathrm{Th}-\mathrm{U}-\mathrm{Pb}$ monazite dating. Estimates of the metamorphic conditions of the granulites based on conventional geothermobarometry and THERMOCALC resulted in temperatures around $800{ }^{\circ} \mathrm{C}$ and pressures between of 5.0 and $9.9 \mathrm{kbar}$ and a retrometamorphic path characterized by near-isobaric cooling. Part of the granulites was affected by anatexis. The melting of felsic granulites resulted in the generation of pegmatites and two aluminous lithotypes. These are:

i) garnet-sillimanite granulite with euhedral plagioclase and cordierite that show straight faces against quartz, and is the crystallization product of an anatectic melt, and

ii) garnet-kyanite-cordierite granulite, which is probably the restite of anatexis, as indicated by textures and high magnesium contents. Th-U-Pb monazite geochronology of two granulite samples resulted in a metamorphic age around $2060 \mathrm{Ma}$, which is similar to the age of the MC registered in the literature. The similar Paleoproterozoic metamorphic ages of both complexes lead to the conclusion that the Acaiaca Complex may be the high grade metamorphic unit geochronologically related to the lower grade Mantiqueira Complex.
\end{abstract}

Keywords: Acaiaca Complex; Granulite; Th-U-Pb monazite dating; Geothermobarometry; Minas Gerais, Brazil.

This strip (Figure 1) extends for at least $36 \mathrm{~km}$ in the north-south direction and has a width of about $6 \mathrm{~km}$. The strip occurs in the central portion of the complex (Medeiros Júnior, 2009; Medeiros Júnior and Jordt-Evangelista, 2010). The identification and description of the AC by Jordt-Evangelista (1984, 1985) and Jordt-Evangelista and Müller (1986a, 1986 b) included ortho-derived mafic and felsic granulites, as well as paraderived kyanite-cordierite-biotite schists. Medeiros Junior and Jordt-Evangelista (2010) described para-derived granulites 
with garnet + sillimanite + quartz + plagioclase + biotite \pm cordierite \pm potassic feldspar, and a metaultramafic granulite composed of olivine and orthopyroxene. Lithotypes derived from sedimentary protoliths have not been described for the Mantiqueira Complex in the region. Metamorphic ages of 2085 to $2041 \mathrm{Ma}$ were obtained by Noce et al. (2007) for the Mantiqueira Complex. The only age published for the AC to date is a Rb-Sr isochron of $1991 \pm 42 \mathrm{Ma}$ (Teixeira et al., 1987), attributed to the granulite facies metamorphism of a felsic granulite.

The discrepancy in metamorphic grades between the Acaiaca and the Mantiqueira complexes, and the existence of lithotypes derived from

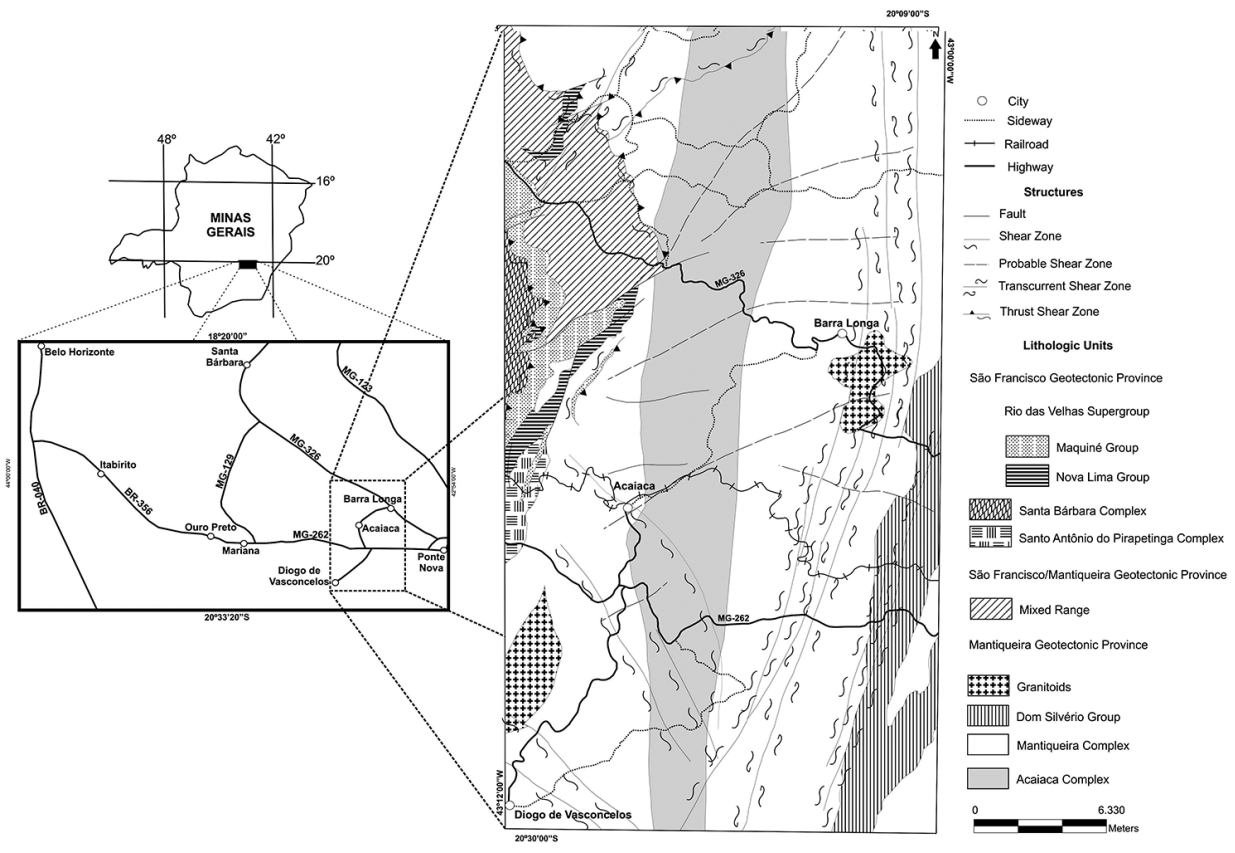

sedimentary protoliths in the AC, led to the present study aiming to understand the metamorphic evolution of the AC. One of the objectives was also to establish whether the granulite facies metamorphism of the Acaiaca Complex was synchronous to the metamorphism of the Mantiqueira Complex by means of Th-U-Pb monazite chemical dating.

\section{Materials and methods}

The study of selected lithotypes from the Acaiaca Complex was based on petrographic and microstructural characterization, mineral chemistry, geothermobarometric calculations and $\mathrm{Th}-\mathrm{U}-\mathrm{Pb}$ dating of metamorphic monazite using electron microprobe (EMP-monazite dating). Quantitative mineral analyses were obtained in the Laboratório de Microanálises at UFMG/ CDTN/CNEN using an electron microprobe JEOL JXA-8900RL, at $15 \mathrm{kV}$ and a beam current of $20 \mathrm{nA}$. The geothermobarometric calculations for mafic and aluminous granulites were performed based on the mineral chemistry data obtained with the electron microprobe. The orthopyroxene-clinopyroxene (Kretz, 1982), hornblende-plagioclase (Holland and Blundy, 1994) and garnet-cordierite (Bhattacharya et al., 1988) geothermometers were used for the estimates of temperature. The plagioclasegarnet-quartz- $\mathrm{Al}_{2} \mathrm{SiO}_{5}$ geobarometer (Newton and Haselton, 1981; Koziol and Newton, 1988; Koziol, 1989) and garnet-cordierite-quartz- $\mathrm{Al}_{2} \mathrm{SiO}_{5}$ geobarometer (Thompson, 1976; Holdaway and Lee, 1977; Wells, 1979) were used for the estimates of pressure. In addition, the conditions of temperature and pressure were calculated by means of the THERMOCALC software, version 3.33 (Powell and Holland, 1994). Two granulite samples were selected for monazite geochronology. These are:

(1) RD01B, felsic granulite with mylonitic features, UTM 696197/ 7743095 , and

(2) RD02A, cordierite-bearing para-derived granulite, UTM 695520/7742163. Analyses of Th, U and $\mathrm{Pb}$ in monazite grains were performed at the Institut für Werkstoffwissenschaft, Freiberg/Germany, with an electron microprobe JEOL JXA$8900 \mathrm{RL}$, using an acceleration voltage of $20 \mathrm{kV}$ and a beam current of $150 \mathrm{nA}$. Counting times for the $5 \mu \mathrm{m}$-diameter beam were $320 \mathrm{~s}(\mathrm{~Pb}), 50 \mathrm{~s}(\mathrm{U})$ and $40 \mathrm{~s}$ (Th). Additionally, the elements La, Y, Ce, Pr, Sm, Nd, Gd, P, Si and Ca were
Figure 1

Geographic location

and geological map of the studied

region, modified from Baltazar and

Raposo (1993), with the areal distribution of the Acaiaca Complex proposed by Medeiros Junior and Jordt-Evangelista (2010).

measured. The calibration of $\mathrm{PbO}$ was carried out on vanadinite standard. A $5 \mathrm{wt} \%$ glass standard was used for calibration of $\mathrm{U}$. The standard used during runs was the monazite Madmon, dated at $496 \pm 9 \mathrm{Ma}$ (U-Pb SHRIMP; Schulz et al., 2007). This standard contains around $10 \mathrm{wt} \% \mathrm{ThO}_{2}$ and was used for offline re-calibration of $\mathrm{ThO}_{2}$ and for data control. Orthophosphates of the Smithsonian Institution (Jarosewich and Boatner, 1991; Donovan et al., 2003) were used as standards for the Rare Earth Elements analyses. The monazite data for each sample was compared in the $\mathrm{ThO}_{2}{ }^{*}-\mathrm{PbO}$ diagram as mentioned in Suzuki et al. (1994). This step allows the calculation of " $i n$ situ" monazite chemical age composed by several analyses, and regression is forced through zero (Montel et al., 1996). The ages given by the slopes of the isochrones coincide with the weighted average ages with a $2 \sigma$ error (Ludwig, 2001). $\mathrm{ThO}_{2}{ }^{*}$ is the sum of the measured $\mathrm{ThO}_{2}$ plus $\mathrm{ThO}_{2}$ equivalent to the measured $\mathrm{UO}_{2}$. 


\section{Petrography}

Based on the recommendations of the International Union of Geological Sciences for the nomenclature of metamorphic rocks (Fettes and Desmons, 2007), the studied samples are classified as felsic granulite, mafic granulite, ultramafic granulite and aluminous granulite. All these rocks, except the last one, show mineral associations typical of ortho-derived rocks. The aluminous rock types have mineral associations typical of pelitic protoliths. Other rock types found in the area of the AC are amphibolite facies biotite and/or hornblende-bearing quartz-feldspathic gneisses, amphibolites, meta-granites, pegmatites and diabase dykes. The pegmatites possibly repre-

Figure 2

Banded granulite

intruded by granitic pegmatite and

diabase dike. Abandoned quarry near Acaiaca (UTM 696197/ 7743095), photograph taken in 1982 by Jordt-Evangelista.

Mafic granulites are constituted of orthopyroxene + clinopyroxene + plagioclase \pm hornblende. Plagioclase has large compositional variation from andesine $\left(A n_{40}\right)$ to bytownite $\left(\mathrm{An}_{87}\right)$. It occurs as xenoblastic grains showing interlobate to polygonal contacts. Hornblende presents greenish-brown to dark green pleochroism and is characterized by color zoning (brown core and greenish edges, Figure 3b). According to the classification of Leake $e t$ al. (1997), this amphibole is classified as Mg-hornblende when $(\mathrm{ANa}+\mathrm{AK})<0.5$, or edenite to Fe-pargasite when $(\mathrm{ANa}+\mathrm{AK})$ $\geq 0.5$. Pyroxenes were often replaced by bluish-green hornblende along the border. Clinopyroxene is classified as diopside. The orthopyroxene composition varies between 32 and $67 \%$ of the enstatite component. It may be partially replaced by cummingtonite. Biotite is strongly pleochroic from red-brown to pale yellow. Garnet is a late metamorphic mineral, because it sent anatectic portions of the granulites (Medeiros Junior, 2009; Medeiros Junior and Jordt-Evangelista, 2010), while the diabases are younger (Figure 2). Locally, the gneisses and also part of the granulites exhibit a mylonitic foliation NNESSW dipping $65^{\circ}$ SEE. The associated gneisses were probably derived from the granulites by lower grade metamorphism accompanying the deformation during exhumation. This relationship is indicated by thrust shear zones characterized by tectonic transport to the west. Felsic and mafic granulites often occur in centimeter to decimeter-wide alternating bands (Figure 2). The felsic granulites are comprised of biotite + plagioclase + quartz \pm potassic feldspar \pm garnet \pm orthopyroxene. Biotite is reddish-brown to pale yellow and contains apatite and zircon inclusions. Plagioclase is oligoclase $\left(\mathrm{An}_{20}-\mathrm{An}_{30}\right)$ and often occurs as antiperthitic xenoblasts. Quartz occurs as xenoblastic grains with strong undulatory extinction (Figure 3a). The potassic feldspar only shows Tartan twinning in strongly deformed portions. Orthopyroxene may be partially replaced by hornblende (Figure 3a). Garnet occurs as porphyroblasts with quartz, biotite, and more rarely feldspar inclusions. Garnet is composed of 70 and $80 \%$ almandine component, 15 to $20 \%$ pyrope, while grossular and spessartite make up 5\% each.

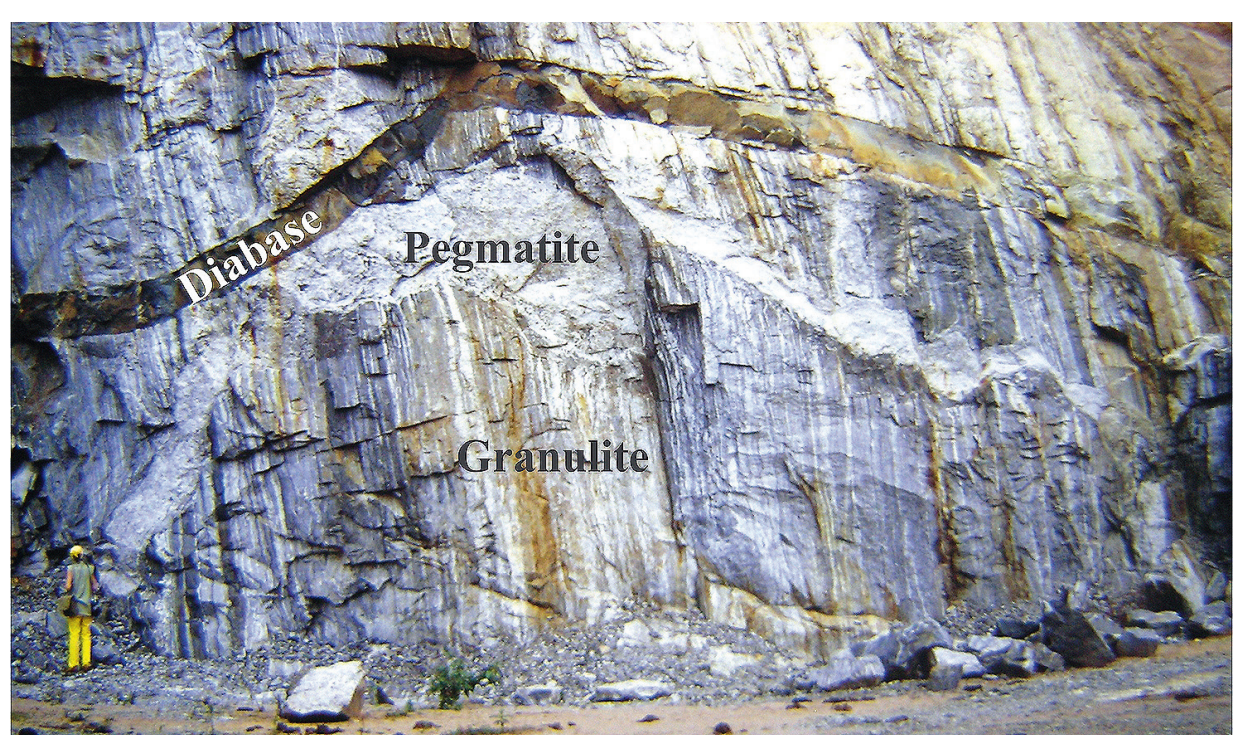

often occurs as coronas on pyroxene and plagioclase (Figure 3c) or as symplectitic intergrowths with ilmenite. Garnet is more calcic and less rich in iron and magnesium, with almandine component around $60 \%$, pyrope $16 \%$, grossular $20 \%$ and spessartite $4 \%$.

The harzburgitic ultramafic granulite consists mostly of olivine and orthopyroxene (Figure $3 \mathrm{~d}$ ) as granoblastic to poiquiloblastic grains reaching up to $2 \mathrm{~cm}$. Orthopyroxene may be partially replaced by carbonate, talc, and anthophyllite. Pyroxene is magnesian, with the enstatite content around $90 \%$. Olivine is forsterite $\left(\mathrm{Fo}_{92}-\mathrm{Fo}_{96}\right)$ and can display replacement by talc, anthophyllite and serpentine.

The aluminous granulites are derived from pelitic sedimentary rocks as indicated by the presence of $\mathrm{Al}_{2} \mathrm{SiO}_{5}$ polymorphs. Based on the type of $\mathrm{Al}_{2} \mathrm{SiO}_{5}$ polymorph and on textural aspects, the granulites can be grouped into garnet-sillimanite granulite and garnet-cordierite-kyanite schist. The first type presents a granoblastic texture and is made up of garnet + biotite + sillimanite + plagioclase + quartz \pm potassic feldspar \pm cordierite. The anhedral to subhedral plagioclase is oligoclase $\left(\mathrm{An}_{21}{ }^{-}\right.$ $\mathrm{An}_{30}$ ) and can be antiperthitic. Quartz often exhibits strong undulatory extinction. Cordierite occurs as idioblastic grains exhibiting square sections (Figure 3e). Potassic feldspar is anhedral to subhedral and commonly microperthitic. Garnet constitutes xenoblastic to subidioblastic porphyroblasts with quartz, biotite and plagioclase inclusions. It is composed of 60 to $80 \%$ almandine component, 15 to $30 \%$ pyrope around $5 \%$ grossular and up to $4 \%$ spessartite. Both biotite and sillimanite are commonly rimming cordierite, feldspar and garnet. Biotite is reddish brown and may contain inclusions of rutile, zircon and apatite. Sillimanite usually occurs in the form of fibrolite intergrowth with biotite. 
The second type of aluminous granulite is a schist with microstructures indicative of disequilibrium, as shown by the partial replacement of an older generation of kyanite, plagioclase, garnet, quartz, biotite, and staurolite by poiquiloblasts of younger cordierite (Figure 3f). Staurolite is a minor mineral found as inclusions in garnet or surrounded by cordierite. Plagioclase is andesine $\left(\mathrm{An}_{31}-\mathrm{An}_{43}\right)$. Quartz has strong undulatory extinction. Biotite is weakly pleochroic in shades of light brown to colorless. It displays preferential orientation and contains rutile, zircon, monazite and apatite inclusions. Magnesium-rich chlorite is the product of the partial replacement of
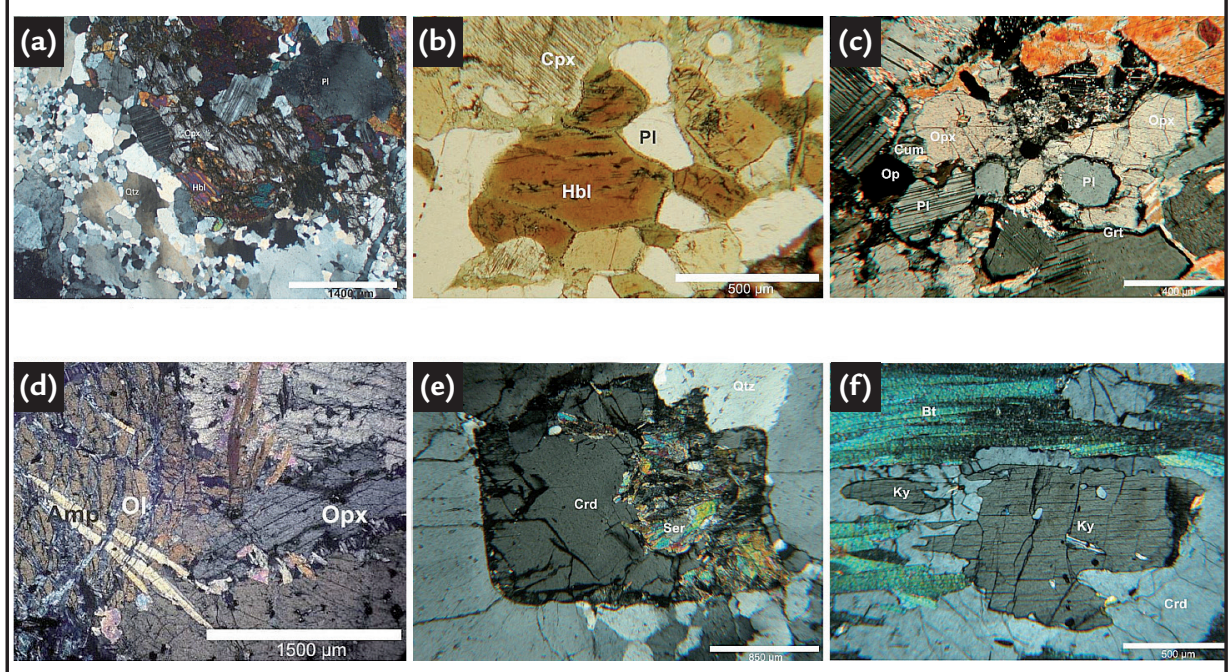

biotite and cordierite. Garnet occurs as xenoblastic to subidioblastic porphyroblasts that contain quartz, biotite, rutile, plagioclase, kyanite and staurolite inclusions. The chemical composition of garnet differs from that found in the garnet-sillimanite granulite in terms of the grossular component that reaches around $10 \%$.

Figure 3

Photomicrographs of selected lithotypes of the Acaiaca Complex.

a) felsic granulite with deformed quartz (Qtz) and orthopyroxene (Opx) partially replaced by hornblende (Hbl) (XPL).

b) hornblende $(\mathrm{Hbl})$ characterized by color zoning (brown core and greenish edges) in mafic granulite (PPL).

c) mafic granulite with orthopyroxene (Opx) partially replaced by cummingtonite (Cum) and with garnet (Grt) as corona on pyroxene and plagioclase (PI) (XPL). d) Harzburgitic ultramafic granulite displaying olivine grains $(\mathrm{Ol})$, orthopyroxene (Opx) and partially replacement by anthophyllite (Amp) (XPL).

e) Garnet-sillimanite granulite with nearly square section of cordierite (Crd) (XPL). f) Kyanite (Ky) surrounded by cordierite (Crd) in garnet-kyanite-cordierite schist (XPL). PPL: plane polarized light; XPL: crossed polarized light.

\section{Geothermobarometry}

The granulite facies mineral association plagioclase + orthopyroxene + clinopyroxene \pm hornblende of the mafic granulites is characteristic of relatively low pressure conditions (De Ward, 1965). The application of the orthopyroxene-clinopyroxene geothermometer (Kretz, 1982) resulted in temperatures up to $745^{\circ} \mathrm{C}$, while the plagioclaseamphibole geothermometer (Holland $\&$ Blundy 1994 ) resulted in $848^{\circ} \mathrm{C}$, assuming a pressure of $5 \mathrm{kbar}$. By means of the software THERMOCALC (Powell and Holland, 1994), it was possible to calculate the temperature and pressure conditions most likely to have generated these rocks. The chemical data of the core of the mineral phases provided $768 \pm 28^{\circ} \mathrm{C}$ and $9.9 \pm 1.6 \mathrm{kbar}$. The rim data indicated $761 \pm 28^{\circ} \mathrm{C}$ and $9.8 \pm 1.5$ kbar, both calculations considering a mole fraction of $\mathrm{H}_{2} \mathrm{O}$ of 0.1 .

\section{Electron microprobe monazite dating}

The results of the EMP-dating of monazite from the two granulite
The mineral association that probably characterizes the peak of progressive metamorphism in the garnet-sillimanite granulite is given by garnet + plagioclase + quartz + sillimanite + biotite \pm potassic feldspar \pm cordierite. Calculations based on the garnet-cordierite geothermometer of Bhattacharya et al. (1988) resulted in temperatures around $694^{\circ} \mathrm{C}$ considering a pressure of $5 \mathrm{kbar}$. For estimation of the pressure conditions, two geobarometers were used, the garnet-sillimanitequartz-plagioclase (GASP) and the garnet-cordierite-quartz- $\mathrm{Al}_{2} \mathrm{SiO}_{5}$. The first resulted in a pressure of $4.9 \mathrm{kbar}$ for the calibration of Newton and Haselton (1981), 5.8 kbar for the Koziol and Newton (1988) and $6.7 \mathrm{kbar}$ for the Koziol (1989) calibration, considering a temperature of $750^{\circ} \mathrm{C}$. The geobarometer garnet-cordierite-quartz- $\mathrm{Al}_{2} \mathrm{SiO}_{5}$ yielded, under the same conditions of temperature, relatively higher pressures of $7.1 \mathrm{kbar}$ for the Thompson (1976) calibration, $8.3 \mathrm{kbar}$ for the Holdaway and Lee (1977), and $7.5 \mathrm{kbar}$ for the Wells (1979) calibration. Calculations by the software THERMOCALC (Powell and Holland, 1994) for the core of the mineral phases of the main association resulted in $712 \pm 79^{\circ} \mathrm{C}$ and $5.0 \pm 0.7$ kbar, assuming a molar fraction of $\mathrm{H}_{2} \mathrm{O}$ of 0.1. Conventional geothermobarometry provided lower temperatures of 630 $716^{\circ} \mathrm{C}$ (pressures of $4.9-8.3 \mathrm{kbar}$ ) while THERMOCALC resulted in $712 \pm 79^{\circ} \mathrm{C}$ and $5 \pm 0.7 \mathrm{kbar}$. The large errors for the results of THERMOCALC suggest disequilibrium during the retrometamorphic process. The geothermobarometric calculations for mafic and aluminous granulites were performed based on the mineral chemistry data shown in Tables 1,2 and 3 .

in the granoblastic matrix and less often also enclosed in garnet porphyroblasts. samples are displayed on Table 4. The monazite grains in both samples occur 
They have maximum lengths of 100 $\mu \mathrm{m}$ which allowed up to 6 single spot analyses within a grain. No significant graytone zonations were detected in the backscattered electron signals of the large grains. Systematic zonations from older cores to younger rims are absent.

Figure 4

Th-U-Pb model ages of monazites in the Acaiaca granulites. RD01B: ortho-derived felsic granulite; RD02A: cordierite-bearing para-derived granulite. Total $\mathrm{PbO}$ vs.

$\mathrm{ThO}_{2}{ }^{*}$ (wt\%) isochron diagrams. $\mathrm{ThO}_{2}{ }^{*}$

is $\mathrm{ThO}_{2}+\mathrm{UO}_{2}$ equivalents expressed as

$\mathrm{ThO}_{2}$. Abbreviations: $\mathrm{Mnz}=$ monazite, Grt=garnet. Madmon is the monazite standard used during runs (Schulz et al., 2007).
The results revealed only one generation of monazite in both granulite samples. The weighted average ages of the matrix grains are $2063 \pm 10 \mathrm{Ma}$ for $\mathrm{RD} 01 \mathrm{~B}$, and $2069 \pm 15 \mathrm{Ma}$ for RD02A (Figure 4). Monazite enclosed in garnet displays slightly older ages than

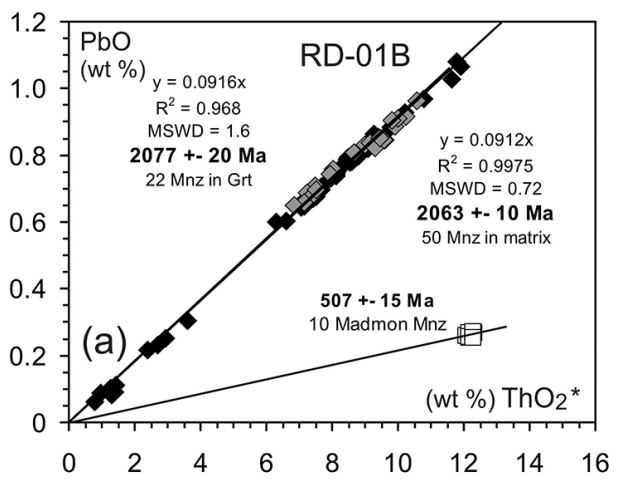

the bulk of the matrix grains, yielding $2077 \pm 20 \mathrm{Ma}$ (RD01B; 22 grains) and $2148 \pm 48$ (RD02A; 4 grains) thus suggesting that the garnet porphyroblasts are older than the matrix. Both groups of monazites belong to a single isochron (Figure 4).

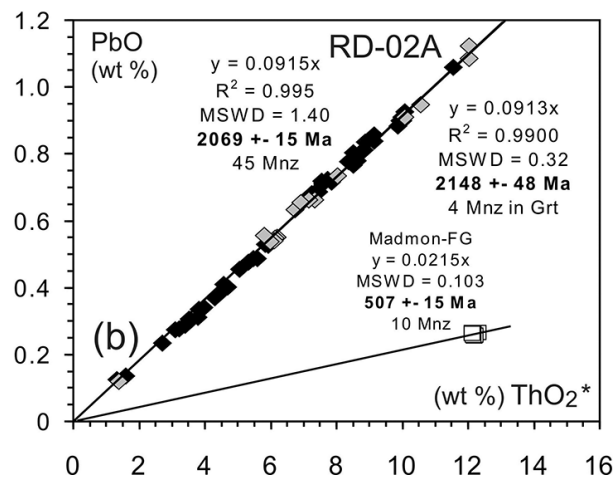

Table 1

Chemical composition (weight \%) of selected minerals in mafic granulite (sample 76J) and their cationic distribution.

\begin{tabular}{|c|c|c|c|c|c|c|c|c|c|c|c|c|c|c|c|c|}
\hline & \multicolumn{3}{|c|}{ Orthopyroxene } & \multicolumn{4}{|c|}{ Clinopyroxene } & \multicolumn{4}{|c|}{ Hornblende } & \multicolumn{5}{|c|}{ Plagioclase } \\
\hline & \multicolumn{6}{|c|}{6 Oxygen } & \multicolumn{6}{|c|}{23 Oxygen } & \multicolumn{4}{|c|}{32 Oxygen } \\
\hline & Opx1 & Opx1 & Cpx1 & Cpx2 & Cpx2 & Cpx2 & & $\mathrm{Hbl} 1$ & $\mathrm{Hbl} 1$ & $\mathrm{Hbl} 2$ & $\mathrm{Hbl} 2$ & & $\mathrm{Pl} 1$ & $\mathrm{Pl} 1$ & $\mathrm{Pl} 2$ & $\mathrm{Pl} 2$ \\
\hline & Rim1 & Core2 & Core3 & Rim1 & Core1 & Core2 & & Core & Rim & Core & Rim & & Rim & Core & Rim & Core \\
\hline $\mathrm{SiO}_{2}$ & 53.55 & 54.29 & 53.94 & 53.51 & 52.95 & 53.04 & $\mathrm{SiO}_{2}$ & 45.03 & 46.20 & 45.76 & 45.86 & $\mathrm{SiO}_{2}$ & 48.26 & 49.35 & 50.88 & 50.17 \\
\hline $\mathrm{TiO}_{2}$ & 0.04 & 0.04 & 0.09 & 0.08 & 0.09 & 0.13 & $\mathrm{TiO}_{2}$ & 2.91 & 1.44 & 1.77 & 1.59 & $\mathrm{TiO}_{2}$ & 0.00 & 0.02 & 0.00 & 0.00 \\
\hline $\mathrm{Al}_{2} \mathrm{O}_{3}$ & 0.52 & 0.72 & 0.73 & 0.66 & 1.17 & 1.10 & $\mathrm{Al}_{2} \mathrm{O}_{3}$ & 9.54 & 10.12 & 10.33 & 10.31 & $\mathrm{Al}_{2} \mathrm{O}_{3}$ & 32.96 & 32.29 & 31.30 & 32.06 \\
\hline $\mathrm{FeO}$ & 21.72 & 21.97 & 6.97 & 6.85 & 7.36 & 6.95 & $\mathrm{FeO}$ & 10.01 & 9.71 & 9.55 & 9.66 & $\mathrm{FeO}$ & 0.03 & 0.00 & 0.09 & 0.03 \\
\hline $\mathrm{Cr}_{2} \mathrm{O}_{3}$ & 0.05 & 0.00 & 0.01 & 0.04 & 0.09 & 0.05 & $\mathrm{Cr}_{2} \mathrm{O}_{3}$ & 0.33 & 0.28 & 0.28 & 0.28 & $\mathrm{MnO}$ & 0.00 & 0.05 & 0.01 & 0.00 \\
\hline $\mathrm{MnO}$ & 0.46 & 0.43 & 0.19 & 0.23 & 0.22 & 0.23 & $\mathrm{MnO}$ & 0.09 & 0.19 & 0.11 & 0.11 & $\mathrm{CaO}$ & 15.80 & 15.17 & 13.82 & 14.69 \\
\hline $\mathrm{MgO}$ & 22.55 & 22.76 & 13.92 & 14.04 & 13.98 & 13.99 & $\mathrm{MgO}$ & 14.03 & 13.85 & 13.84 & 13.63 & $\mathrm{Na}_{2} \mathrm{O}$ & 2.14 & 2.52 & 3.08 & 2.70 \\
\hline $\mathrm{CaO}$ & 0.28 & 0.30 & 23.45 & 23.61 & 22.42 & 22.42 & $\mathrm{CaO}$ & 11.45 & 11.79 & 11.76 & 11.75 & $\mathrm{~K}_{2} \mathrm{O}$ & 0.04 & 0.06 & 0.11 & 0.10 \\
\hline $\mathrm{Na}_{2} \mathrm{O}$ & 0.03 & 0.00 & 0.22 & 0.16 & 0.24 & 0.22 & $\mathrm{Na}_{2} \mathrm{O}$ & 1.32 & 1.28 & 1.32 & 1.44 & Total & 99.22 & 99.46 & 99.31 & 99.77 \\
\hline $\mathrm{K}_{2} \mathrm{O}$ & 0.01 & 0.02 & 0.01 & 0.00 & 0.00 & 0.01 & $\mathrm{~K} 2 \mathrm{O}$ & 1.00 & 1.00 & 1.05 & 1.05 & $\mathrm{Si}$ & 8.88 & 9.04 & 9.30 & 9.15 \\
\hline Total & 99.20 & 100.52 & 99.53 & 99.17 & 98.52 & 98.14 & $\mathrm{~F}$ & 0.40 & 0.35 & 0.50 & 0.56 & $\mathrm{Al}$ & 7.14 & 6.97 & 6.74 & 6.88 \\
\hline TSi & 2.01 & 2.01 & 2.01 & 2.00 & 2.00 & 2.01 & $\mathrm{Cl}$ & 0.03 & 0.02 & 0.04 & 0.03 & $\mathrm{Ti}$ & 0.00 & 0.00 & 0.00 & 0.00 \\
\hline TAl & 0.00 & 0.00 & 0.00 & 0.00 & 0.01 & 0.00 & Total & 95.79 & 95.95 & 96.03 & 96.00 & $\mathrm{Fe}^{2}$ & 0.01 & 0.00 & 0.01 & 0.01 \\
\hline M1Al & 0.02 & 0.03 & 0.03 & 0.03 & 0.05 & 0.05 & TSi & 6.69 & 6.83 & 6.78 & 6.81 & $\mathrm{Mn}$ & 0.00 & 0.01 & 0.00 & 0.00 \\
\hline M1Ti & 0.00 & 0.00 & 0.00 & 0.00 & 0.00 & 0.00 & TAl & 1.31 & 1.17 & 1.22 & 1.19 & $\mathrm{Ca}$ & 3.12 & 2.98 & 2.71 & 2.87 \\
\hline $\mathrm{M} 1 \mathrm{Fe}^{2}$ & 0.00 & 0.00 & 0.19 & 0.18 & 0.16 & 0.16 & CAl & 0.36 & 0.59 & 0.58 & 0.62 & $\mathrm{Na}$ & 0.76 & 0.90 & 1.09 & 0.96 \\
\hline $\mathrm{M} 1 \mathrm{Cr}$ & 0.00 & 0.00 & 0.00 & 0.00 & 0.00 & 0.00 & $\mathrm{CCr}$ & 0.04 & 0.03 & 0.03 & 0.03 & K & 0.01 & 0.01 & 0.03 & 0.02 \\
\hline $\mathrm{M} 1 \mathrm{Mg}$ & 0.98 & 0.97 & 0.77 & 0.78 & 0.79 & 0.79 & $\mathrm{CFe}^{3}$ & 0.09 & 0.02 & 0.00 & 0.00 & Albite & 19.60 & 23.00 & 28.50 & 24.80 \\
\hline $\mathrm{M} 2 \mathrm{Mg}$ & 0.29 & 0.29 & 0.00 & 0.00 & 0.00 & 0.00 & $C T i$ & 0.33 & 0.16 & 0.20 & 0.18 & Anortite & 80.20 & 76.60 & 70.70 & 74.60 \\
\hline $\mathrm{M} 2 \mathrm{Fe}^{2}$ & 0.68 & 0.68 & 0.03 & 0.03 & 0.07 & 0.06 & $\mathrm{CMg}$ & 3.11 & 3.05 & 3.06 & 3.02 & Orthoclase & 0.20 & 0.40 & 0.70 & 0.60 \\
\hline $\mathrm{M} 2 \mathrm{Mn}$ & 0.02 & 0.01 & 0.01 & 0.01 & 0.01 & 0.01 & $\mathrm{CFe}^{2}$ & 1.08 & 1.14 & 1.13 & 1.15 & & & & & \\
\hline $\mathrm{M} 2 \mathrm{Ca}$ & 0.01 & 0.01 & 0.94 & 0.95 & 0.91 & 0.91 & CMn & 0.02 & 0.02 & 0.02 & 0.02 & & & & & \\
\hline $\mathrm{M} 2 \mathrm{Na}$ & 0.00 & 0.00 & 0.02 & 0.01 & 0.02 & 0.02 & $\mathrm{BFe}^{2}$ & 0.08 & 0.05 & 0.06 & 0.05 & & & & & \\
\hline M2K & 0.00 & 0.00 & 0.00 & 0.00 & 0.00 & 0.00 & $\mathrm{BCa}$ & 1.82 & 1.87 & 1.87 & 1.87 & & & & & \\
\hline Enstatite & 65 & 65 & - & - & - & - & $\mathrm{BNa}$ & 0.10 & 0.07 & 0.07 & 0.07 & & & & & \\
\hline Ferrosilite & 35 & 35 & - & - & - & - & AK & 0.19 & 0.19 & 0.20 & 0.20 & & & & & \\
\hline & & & & & & & CF & 0.19 & 0.16 & 0.24 & 0.26 & & & & & \\
\hline
\end{tabular}


Table 2

Chemical composition (weight \%) of selected minerals in mafic granulite (samples 3B and 32) and their cationic distribution.

\begin{tabular}{|c|c|c|c|c|c|c|c|c|c|c|c|c|c|}
\hline $\begin{array}{c}\text { Sample } \\
3 B\end{array}$ & \multicolumn{3}{|c|}{ Hornblende } & & \multicolumn{2}{|c|}{ Plagioclase } & $\begin{array}{c}\text { Sample } \\
32\end{array}$ & Orthopyroxene & Clinopyroxene & & Hornblende & & Plagioclase \\
\hline & \multicolumn{3}{|c|}{23 Oxygen } & & \multicolumn{2}{|c|}{32 Oxygen } & & 6 Oxygen & 6 Oxygen & & 23 Oxygen & & 32 Oxygen \\
\hline & $\mathrm{Hbl2}$ & $\mathrm{Hbl} 2$ & $\mathrm{Hbl} 2$ & & Pl1 & $\mathrm{Pl} 1$ & & Opx1 & Cpx2 & & Hbl1 & & $\mathrm{Pl} 1$ \\
\hline & Core1 & Core2 & Rim2 & & Rim & Core & & Core & Core & & Core & & Core \\
\hline $\mathrm{SiO}_{2}$ & 40.98 & 41.25 & 41.01 & $\mathrm{SiO}_{2}$ & 53.22 & 53.26 & $\mathrm{SiO}_{2}$ & 51.58 & 52.62 & $\mathrm{SiO}_{2}$ & 45.46 & $\mathrm{SiO}_{2}$ & 52.96 \\
\hline $\mathrm{TiO}_{2}$ & 2.11 & 2.45 & 0.99 & $\mathrm{Al}_{2} \mathrm{O}_{3}$ & 29.95 & 29.76 & $\mathrm{TiO}_{2}$ & 0.08 & 0.15 & $\mathrm{TiO}_{2}$ & 1.61 & $\mathrm{Al}_{2} \mathrm{O}_{3}$ & 30.37 \\
\hline $\mathrm{Al} 2 \mathrm{O}_{3}$ & 12.65 & 12.41 & 14.38 & $\mathrm{FeO}$ & 0.07 & 0.07 & $\mathrm{Al}_{2} \mathrm{O}_{3}$ & 0.67 & 1.28 & $\mathrm{Al}_{2} \mathrm{O}_{3}$ & 10.50 & $\mathrm{FeO}$ & 0.00 \\
\hline $\mathrm{FeO}$ & 20.27 & 20.52 & 19.71 & $\mathrm{MnO}$ & 0.01 & 0 & $\mathrm{FeO}$ & 31.84 & 11.68 & $\mathrm{FeO}$ & 16.34 & $\mathrm{MnO}$ & 0.00 \\
\hline $\mathrm{Cr}_{2} \mathrm{O}_{3}$ & 0.13 & 0.14 & 0.13 & $\mathrm{CaO}$ & 12.22 & 12.18 & $\mathrm{Cr}_{2} \mathrm{O}_{3}$ & 0.01 & 0.00 & $\mathrm{Cr}_{2} \mathrm{O}_{3}$ & 0.04 & $\mathrm{CaO}$ & 13.19 \\
\hline $\mathrm{MnO}$ & 0.23 & 0.19 & 0.16 & $\mathrm{Na}_{2} \mathrm{O}$ & 4.03 & 4.08 & $\mathrm{MnO}$ & 0.71 & 0.23 & $\mathrm{MnO}$ & 0.10 & $\mathrm{Na}_{2} \mathrm{O}$ & 3.88 \\
\hline $\mathrm{MgO}$ & 6.21 & 6.27 & 6.13 & $\mathrm{~K}_{2} \mathrm{O}$ & 0.1 & 0.07 & $\mathrm{MgO}$ & 15.33 & 11.67 & $\mathrm{MgO}$ & 10.24 & $\mathrm{~K}_{2} \mathrm{O}$ & 0.07 \\
\hline $\mathrm{CaO}$ & 11.43 & 11.49 & 11.66 & Total & 99.61 & 99.42 & $\mathrm{CaO}$ & 0.50 & 22.65 & $\mathrm{CaO}$ & 11.61 & Total & 100.45 \\
\hline $\mathrm{Na}_{2} \mathrm{O}$ & 1.36 & 1.31 & 1.38 & $\mathrm{Si}$ & 9.65 & 9.67 & $\mathrm{Na}_{2} \mathrm{O}$ & 0.00 & 0.24 & $\mathrm{Na}_{2} \mathrm{O}$ & 1.23 & $\mathrm{Si}$ & 9.54 \\
\hline $\mathrm{K}_{2} \mathrm{O}$ & 1.65 & 1.81 & 1.26 & Al & 6.39 & 6.36 & $\mathrm{~K}_{2} \mathrm{O}$ & 0.01 & 0.00 & $\mathrm{~K}_{2} \mathrm{O}$ & 0.79 & $\mathrm{Al}$ & 6.44 \\
\hline $\mathrm{F}$ & 0.07 & 0.04 & 0.11 & $\mathrm{Fe} 2$ & 0.01 & 0.01 & Total & 100.73 & 100.52 & $\mathrm{~F}$ & 0.00 & $\mathrm{Ti}$ & 0.00 \\
\hline $\mathrm{Cl}$ & 0.4 & 0.37 & 0.3 & $\mathrm{Mn}$ & 0 & 0 & TSi & 2.00 & 1.98 & $\mathrm{Cl}$ & 0.09 & $\mathrm{Fe}^{2}$ & 0.00 \\
\hline Total & 97.35 & 98.11 & 97.07 & $\mathrm{Ca}$ & 2.37 & 2.37 & TAl & 0.00 & 0.02 & Total & 97.99 & $\mathrm{Mn}$ & 0.00 \\
\hline TSi & 6.37 & 6.37 & 6.32 & $\mathrm{Na}$ & 1.42 & 1.44 & M1Al & 0.03 & 0.04 & TSi & 6.75 & $\mathrm{Ca}$ & 2.55 \\
\hline TAl & 1.63 & 1.63 & 1.68 & K & 0.02 & 0.02 & M1Ti & 0.00 & 0.00 & TAl & 1.25 & $\mathrm{Na}$ & 1.36 \\
\hline CAl & 0.68 & 0.63 & 0.93 & Cations & 19.87 & 19.87 & $\mathrm{M} 1 \mathrm{Fe}^{2}$ & 0.08 & 0.31 & CAl & 0.58 & K & 0.02 \\
\hline $\mathrm{CFe}^{3}$ & 0 & 0 & 0.02 & Albite & 37.2 & 37.6 & $\mathrm{M} 1 \mathrm{Cr}$ & 0.00 & 0.00 & $\mathrm{CFe}^{3}$ & 0.11 & Albite & 34.6 \\
\hline$C T i$ & 0.25 & 0.29 & 0.12 & Anorthite & 62.2 & 62.0 & $\mathrm{M} 1 \mathrm{Mg}$ & 0.89 & 0.65 & $C T i$ & 0.18 & Anortite & 65 \\
\hline $\mathrm{CMg}$ & 1.44 & 1.44 & 1.41 & Orthoclase & 0.6 & 0.4 & $\mathrm{M} 2 \mathrm{Mg}$ & 0.00 & 0.00 & $\mathrm{CMg}$ & 2.27 & Orthoclase & 0.4 \\
\hline $\mathrm{CFe}^{2}$ & 2.6 & 2.62 & 2.5 & & & & $\mathrm{M} 2 \mathrm{Fe}^{2}$ & 0.95 & 0.06 & $\mathrm{CFe}^{2}$ & 1.85 & & \\
\hline $\mathrm{CMn}$ & 0.02 & 0.01 & 0.01 & & & & $\mathrm{M} 2 \mathrm{Mn}$ & 0.02 & 0.01 & $\mathrm{CMn}$ & 0.01 & & \\
\hline $\mathrm{BFe}^{2}$ & 0.03 & 0.03 & 0.02 & & & & $\mathrm{M} 2 \mathrm{Ca}$ & 0.02 & 0.91 & $\mathrm{BFe}^{2}$ & 0.07 & & \\
\hline $\mathrm{BCa}$ & 1.9 & 1.9 & 1.93 & & & & $\mathrm{M} 2 \mathrm{Na}$ & 0.00 & 0.02 & $\mathrm{BCa}$ & 1.85 & & \\
\hline $\mathrm{ANa}$ & 0.41 & 0.39 & 0.41 & & & & $\mathrm{M} 2 \mathrm{~K}$ & 0.00 & 0.00 & $\mathrm{ANa}$ & 0.43 & & \\
\hline AK & 0.33 & 0.36 & 0.25 & & & & Enstatite & 48 & - & AK & 0.15 & & \\
\hline $\mathrm{CCl}$ & 0.11 & 0.1 & 0.08 & & & & Ferrosilite & 52 & - & $\mathrm{CCl}$ & 0.02 & & \\
\hline
\end{tabular}


Table 3

Chemical composition (weight \%) of selected minerals in aluminous granulite (sample 101) and their cationic distribution.

\begin{tabular}{|c|c|c|c|c|c|c|c|c|c|c|}
\hline & \multicolumn{3}{|c|}{ Cordierite } & & \multicolumn{2}{|c|}{ Garnet } & & \multicolumn{2}{|l|}{ Biotite } & \multirow{2}{*}{$\begin{array}{c}\text { Plagioclase } \\
\text { Pl1 }\end{array}$} \\
\hline & Crd1 & Crd1 & Crd1 & & Grt1 & Grt2 & & Bt1 & & \\
\hline & Core 1 & Rim & Core2 & & Core & Core & & Core & & Core \\
\hline & \multicolumn{3}{|c|}{18 Oxygen } & & \multicolumn{2}{|c|}{12 Oxygen } & & 22 Oxygen & & 32 Oxygen \\
\hline $\mathrm{SiO}_{2}$ & 49.88 & 49.77 & 49.51 & $\mathrm{SiO}_{2}$ & 37.03 & 38.14 & $\mathrm{SiO}_{2}$ & 37.85 & $\mathrm{SiO}_{2}$ & 62.35 \\
\hline $\mathrm{Al}_{2} \mathrm{O}_{3}$ & 33.34 & 33.34 & 33.27 & $\mathrm{TiO}_{2}$ & 0.00 & 0.00 & $\mathrm{TiO}_{2}$ & 4.61 & $\mathrm{TiO}_{2}$ & 0.02 \\
\hline $\mathrm{FeO}$ & 4.48 & 5.00 & 4.96 & $\mathrm{Al}_{2} \mathrm{O}_{3}$ & 24.29 & 22.37 & $\mathrm{Al}_{2} \mathrm{O}_{3}$ & 16.69 & $\mathrm{Al}_{2} \mathrm{O}_{3}$ & 24.19 \\
\hline $\mathrm{MnO}$ & 0.02 & 0.00 & 0.03 & $\mathrm{FeO}$ & 31.22 & 33.48 & $\mathrm{Cr}_{2} \mathrm{O}_{3}$ & 0.13 & $\mathrm{FeO}$ & 0.00 \\
\hline $\mathrm{MgO}$ & 10.44 & 9.84 & 9.96 & $\mathrm{MnO}$ & 0.24 & 0.63 & $\mathrm{FeO}$ & 15.11 & $\mathrm{MnO}$ & 0.00 \\
\hline $\mathrm{CaO}$ & 0.03 & 0.01 & 0.00 & $\mathrm{MgO}$ & 7.41 & 5.85 & $\mathrm{MnO}$ & 0.01 & $\mathrm{CaO}$ & 5.38 \\
\hline $\mathrm{Na}_{2} \mathrm{O}$ & 0.02 & 0.04 & 0.04 & $\mathrm{CaO}$ & 0.39 & 0.85 & $\mathrm{MgO}$ & 13.35 & $\mathrm{Na}_{2} \mathrm{O}$ & 7.58 \\
\hline $\mathrm{K}_{2} \mathrm{O}$ & 0.01 & 0.00 & 0.02 & $\mathrm{Na}_{2} \mathrm{O}$ & 0.01 & 0.02 & $\mathrm{CaO}$ & 0.02 & $\mathrm{~K}_{2} \mathrm{O}$ & 0.28 \\
\hline Total & 98.21 & 97.99 & 97.80 & $\mathrm{~K}_{2} \mathrm{O}$ & 0.00 & 0.00 & $\mathrm{Na} 2 \mathrm{O}$ & 0.04 & Total & 99.78 \\
\hline $\mathrm{Si}$ & 5.04 & 5.05 & 5.04 & Total & 100.59 & 101.34 & $\mathrm{~K} 2 \mathrm{O}$ & 8.43 & $\mathrm{Si}$ & 11.03 \\
\hline $\mathrm{Al}$ & 3.97 & 3.98 & 3.99 & TSi & 2.86 & 2.97 & $\mathrm{~F}$ & 0.62 & $\mathrm{Al}$ & 5.04 \\
\hline $\mathrm{Ti}$ & 0.00 & 0.00 & 0.00 & TAl & 0.14 & 0.03 & $\mathrm{Cl}$ & 0.60 & $\mathrm{Ti}$ & 0.00 \\
\hline $\mathrm{Fe}^{2}$ & 0.38 & 0.42 & 0.42 & AIVI & 2.08 & 2.02 & Total & 97.46 & $\mathrm{Fe}^{2}$ & 0.00 \\
\hline $\mathrm{Mn}$ & 0.00 & 0.00 & 0.00 & $\mathrm{Ti}$ & 0.00 & 0.00 & $\mathrm{Si}$ & 5.29 & $\mathrm{Mn}$ & 0.00 \\
\hline $\mathrm{Mg}$ & 1.57 & 1.49 & 1.51 & $\mathrm{Fe}^{2}$ & 2.02 & 2.18 & AlIV & 2.71 & $\mathrm{Ca}$ & 1.02 \\
\hline $\mathrm{Ca}$ & 0.00 & 0.00 & 0.00 & $\mathrm{Mg}$ & 0.85 & 0.68 & AlVI & 0.04 & $\mathrm{Na}$ & 2.60 \\
\hline $\mathrm{Na}$ & 0.00 & 0.01 & 0.01 & $\mathrm{Mn}$ & 0.02 & 0.04 & $\mathrm{Ti}$ & 0.49 & $\mathrm{~K}$ & 0.06 \\
\hline K & 0.00 & 0.00 & 0.00 & $\mathrm{Ca}$ & 0.03 & 0.07 & $\mathrm{Fe}^{2}$ & 1.77 & Albite & 70.6 \\
\hline Mg-Crd & 80.5 & 78 & 78.2 & $\mathrm{Na}$ & 0.00 & 0.00 & $\mathrm{Mg}$ & 2.78 & Anortite & 27.7 \\
\hline $\mathrm{Fe}-\mathrm{Crd}$ & 19.5 & 22 & 21.8 & $\mathrm{~K}$ & 0.00 & 0.00 & $\mathrm{Na}$ & 0.01 & Orthoclase & 1.7 \\
\hline & & & & Almadine & 69.2 & 73.4 & K & 1.50 & & \\
\hline & & & & Grossular & 1.0 & 2.4 & $\mathrm{CF}$ & 0.55 & & \\
\hline & & & & Pyrope & 29.1 & 22.9 & $\mathrm{CCl}$ & 0.28 & & \\
\hline & & & & Spessartine & 0.7 & 1.3 & Annite & 39 & & \\
\hline & & & & & & & Phlogopite & 61 & & \\
\hline
\end{tabular}


Table 4

Electron microprobe analyses of metamorphic monazite from ortho-and para-derived granulites from the Acaiaca Complex.

\begin{tabular}{|c|c|c|c|c|c|c|c|c|c|c|c|c|c|c|c|c|c|c|c|c|}
\hline Monazite & $\mathrm{P}_{2} \mathrm{O}_{5}$ & $\mathrm{SiO}_{2}$ & $\mathrm{CaO}$ & $\mathrm{Y}_{2} \mathrm{O}_{3}$ & $\mathrm{La}_{2} \mathrm{O}_{3}$ & $\mathrm{Ce}_{2} \mathrm{O}_{3}$ & $\mathrm{Pr}_{2} \mathrm{O}_{3}$ & $\mathrm{Sm}_{2} \mathrm{O}_{3}$ & $\mathrm{Nd}_{2} \mathrm{O}_{3}$ & $\mathrm{Gd}_{2} \mathrm{O}_{3}$ & $\mathrm{ThO}_{2}$ & $\mathrm{UO}_{2}$ & $\mathrm{PbO}$ & Total & Th & $\cup$ & $\mathrm{Pb}$ & Th* & Age & \pm \\
\hline RD-01B-mz2-4 & 29.08 & 0.51 & 1.55 & 0.30 & 14.15 & 28.33 & 3.21 & 2.20 & 12.90 & 1.44 & 6.68 & 0.38 & 0.742 & 101.49 & 5.874 & 0.332 & 0.689 & 7.144 & 2068 & 72 \\
\hline RD-01B-mz4-g1 & 30.19 & 0.23 & 1.52 & 2.32 & 13.82 & 27.18 & 3.05 & 1.97 & 12.08 & 1.61 & 5.02 & 1.04 & 0.831 & 100.86 & 4.409 & 0.913 & 0.771 & 7.919 & 2093 & 65 \\
\hline RD-01B-mz4-g2 & 29.89 & 0.24 & 1.54 & 2.33 & 13.87 & 27.05 & 3.04 & 1.99 & 12.07 & 1.62 & 5.20 & 1.03 & 0.839 & 100.71 & 4.569 & 0.907 & 0.778 & 8.050 & 2078 & 64 \\
\hline RD-01B-mz4-g3 & 29.93 & 0.27 & 1.34 & 1.92 & 14.09 & 27.82 & 3.15 & 2.04 & 12.42 & 1.61 & 4.93 & 0.65 & 0.693 & 100.85 & 4.332 & 0.573 & 0.643 & 6.537 & 2112 & 79 \\
\hline RD-01B-mz5-g2 & 29.27 & 0.46 & 1.40 & 0.90 & 13.95 & 28.10 & 3.28 & 2.34 & 12.76 & 1.63 & 5.05 & 1.27 & 0.888 & 101.30 & 4.436 & 1.122 & 0.824 & 8.719 & 2034 & 59 \\
\hline RD-01B-mz5-g4 & 29.74 & 0.35 & 1.47 & 0.95 & 13.98 & 28.31 & 3.19 & 2.18 & 12.77 & 1.54 & 4.83 & 1.35 & 0.911 & 101.58 & 4.244 & 1.191 & 0.846 & 8.807 & 2066 & 59 \\
\hline RD-01B-mz6-1 & 29.42 & 0.43 & 1.28 & 0.85 & 14.23 & 28.82 & 3.27 & 2.21 & 12.88 & 1.49 & 5.34 & 0.33 & 0.602 & 101.16 & 4.696 & 0.288 & 0.559 & 5.797 & 2068 & 89 \\
\hline RD-01B-mz9-g-1 & 29.65 & 0.18 & 1.30 & 2.48 & 13.98 & 27.62 & 3.14 & 1.97 & 12.60 & 1.71 & 4.26 & 0.77 & 0.689 & 100.33 & 3.747 & 0.675 & 0.640 & 6.362 & 2156 & 81 \\
\hline RD-01B-mz11-3 & 28.75 & 1.01 & 1.47 & 1.98 & 12.85 & 26.34 & 3.05 & 2.17 & 11.89 & 1.89 & 8.02 & 0.57 & 0.929 & 100.92 & 7.044 & 0.506 & 0.863 & 8.981 & 2061 & 57 \\
\hline RD-01B-mz12-g3 & 28.85 & 0.67 & 1.26 & 0.39 & 14.00 & 28.49 & 3.35 & 2.35 & 13.28 & 1.70 & 6.32 & 0.31 & 0.695 & 101.68 & 5.555 & 0.276 & 0.645 & 6.615 & 2091 & 78 \\
\hline RD-01B-mz12-g4 & 28.83 & 0.81 & 1.47 & 1.12 & 13.08 & 27.04 & 3.19 & 2.33 & 12.81 & 1.85 & 7.37 & 0.64 & 0.905 & 101.44 & 6.474 & 0.561 & 0.840 & 8.628 & 2089 & 60 \\
\hline RD-01B-mz13-1 & 29.27 & 0.57 & 1.33 & 1.72 & 13.72 & 27.46 & 3.17 & 2.22 & 12.29 & 1.96 & 6.44 & 0.22 & 0.665 & 101.03 & 5.662 & 0.198 & 0.618 & 6.420 & 2062 & 80 \\
\hline RD-01B-mz13-6 & 29.25 & 0.73 & 1.61 & 0.87 & 13.88 & 27.51 & 3.11 & 2.24 & 12.29 & 1.81 & 6.43 & 0.64 & 0.816 & 101.17 & 5.651 & 0.560 & 0.758 & 7.802 & 2084 & 66 \\
\hline RD-01B-mz14-3 & 28.90 & 0.69 & 1.33 & 0.24 & 14.16 & 28.62 & 3.25 & 2.07 & 12.92 & 1.27 & 6.89 & 0.15 & 0.686 & 101.19 & 6.056 & 0.133 & 0.637 & 6.565 & 2079 & 78 \\
\hline RD-01B-mz15-3 & 29.71 & 0.24 & 1.50 & 2.51 & 14.07 & 27.20 & 3.05 & 1.93 & 12.01 & 1.70 & 4.68 & 1.23 & 0.855 & 100.68 & 4.108 & 1.083 & 0.794 & 8.258 & 2068 & 63 \\
\hline RD-01B-mz16-g1 & 30.16 & 0.15 & 1.46 & 2.69 & 14.17 & 27.19 & 3.03 & 1.84 & 12.17 & 1.64 & 4.71 & 0.84 & 0.744 & 100.79 & 4.142 & 0.737 & 0.691 & 6.984 & 2123 & 74 \\
\hline RD-01B-mz17-3 & 29.21 & 0.75 & 1.53 & 0.42 & 13.79 & 27.80 & 3.14 & 2.14 & 12.76 & 1.42 & 7.36 & 0.31 & 0.779 & 101.42 & 6.468 & 0.275 & 0.724 & 7.520 & 2063 & 68 \\
\hline RD-02A-mz1-2 & 28.15 & 1.04 & 1.44 & 0.11 & 12.60 & 28.67 & 3.37 & 2.17 & 13.96 & 1.21 & 7.32 & 0.70 & 0.911 & 101.65 & 6.432 & 0.614 & 0.846 & 8.783 & 2067 & 59 \\
\hline RD-02A-mz1-5 & 29.45 & 0.47 & 1.83 & 1.53 & 16.19 & 28.27 & 2.78 & 1.45 & 10.21 & 1.29 & 6.39 & 0.72 & 0.856 & 101.41 & 5.614 & 0.632 & 0.794 & 8.049 & 2116 & 64 \\
\hline RD-02A-mz1-6 & 28.95 & 0.64 & 1.51 & 0.20 & 14.67 & 29.29 & 3.16 & 1.91 & 12.54 & 1.21 & 6.47 & 0.32 & 0.724 & 101.59 & 5.682 & 0.286 & 0.672 & 6.786 & 2121 & 76 \\
\hline RD-02A-mz1-7 & 29.42 & 0.40 & 1.47 & 1.30 & 15.73 & 29.07 & 2.93 & 1.74 & 11.36 & 1.44 & 5.27 & 0.67 & 0.716 & 101.51 & 4.627 & 0.593 & 0.665 & 6.899 & 2069 & 75 \\
\hline RD-02A-mz1-9 & 28.59 & 0.69 & 1.71 & 0.11 & 13.43 & 28.63 & 3.26 & 2.06 & 13.19 & 1.28 & 7.32 & 0.31 & 0.805 & 101.38 & 6.435 & 0.269 & 0.747 & 7.474 & 2139 & 69 \\
\hline RD-02A-mz1-11 & 29.58 & 0.30 & 2.05 & 2.07 & 16.24 & 27.95 & 2.65 & 1.28 & 9.76 & 1.23 & 6.57 & 0.59 & 0.835 & 101.09 & 5.777 & 0.521 & 0.775 & 7.790 & 2131 & 66 \\
\hline RD-02A-mz1-12 & 29.34 & 0.36 & 2.00 & 1.54 & 16.80 & 28.31 & 2.64 & 1.17 & 9.32 & 1.03 & 6.98 & 0.49 & 0.811 & 100.79 & 6.135 & 0.429 & 0.753 & 7.781 & 2076 & 66 \\
\hline RD-02A-mz7-g4 & 30.13 & 0.18 & 1.65 & 2.68 & 14.21 & 27.65 & 2.88 & 1.86 & 11.71 & 1.64 & 4.54 & 0.91 & 0.735 & 100.77 & 3.991 & 0.801 & 0.683 & 7.061 & 2077 & 73 \\
\hline RD-02A-mz10-1 & 29.19 & 0.63 & 1.81 & 0.62 & 12.58 & 27.70 & 3.23 & 2.22 & 13.56 & 1.71 & 7.27 & 0.38 & 0.804 & 101.71 & 6.389 & 0.335 & 0.746 & 7.673 & 2085 & 67 \\
\hline RD-02A-mz13-g & 29.39 & 0.16 & 1.59 & 0.24 & 14.86 & 29.38 & 3.20 & 2.00 & 13.02 & 1.38 & 4.84 & 0.49 & 0.634 & 101.20 & 4.257 & 0.436 & 0.588 & 5.938 & 2124 & 87 \\
\hline RD-02A-mz15-1 & 27.50 & 1.36 & 1.95 & 0.09 & 12.53 & 27.01 & 3.08 & 1.88 & 12.98 & 0.95 & 10.73 & 0.21 & 1.058 & 101.35 & 9.430 & 0.187 & 0.982 & 10.147 & 2074 & 51 \\
\hline RD-02A-mz16-1 & 28.68 & 0.90 & 1.90 & 0.21 & 13.45 & 27.84 & 3.14 & 1.89 & 12.87 & 1.24 & 8.44 & 0.42 & 0.926 & 101.91 & 7.421 & 0.374 & 0.860 & 8.855 & 2081 & 58 \\
\hline RD-02A-mz17-3 & 29.87 & 0.24 & 1.57 & 1.90 & 15.02 & 28.36 & 3.01 & 1.90 & 11.65 & 1.66 & 4.86 & 0.70 & 0.709 & 101.45 & 4.272 & 0.620 & 0.659 & 6.661 & 2121 & 77 \\
\hline Madmon-avg20 & 24.99 & 3.09 & 0.16 & 0.99 & 7.96 & 25.55 & 3.87 & 4.53 & 16.11 & 2.30 & 10.92 & 0.38 & 0.262 & 101.13 & 9.599 & 0.337 & 0.243 & 10.710 & 506 & 50 \\
\hline
\end{tabular}


Th* is calculated from Th and U after Suzuki et al. (1994). Monazite ages from single analyses are given with 2

\section{Discussion}

In the mafic and some felsic rocks, the presence of orthopyroxene indicates metamorphic conditions of the granulite facies. However, part of the data obtained for the classic geothermometers resulted in temperature values consistent with the amphibolite facies. This is a consequence of the retrogressive sigma error. $\mathrm{mz}$ is a monazite single grain; $\mathrm{mz}-\mathrm{g}$ is monazite grain enclosed in garnet. Data from reference standard monazite
Madmon (Schulz et al. 2007) is mean of 20 single analyses performed during sessions on the Acaiaca samples.

$$
\text { Hornblende }+ \text { quartz }=\text { orthopyroxene }+ \text { clinopyroxene }+ \text { plagioclase }+\mathrm{H}_{2} \mathrm{O}
$$

Blue-green hornblende, cummingtonite and garnet are mineral phases present in some samples of the mafic

Garnet occurs as coronas around pyroxene and plagioclase or forming symplectites with ilmenite. The garnet

The ultramafic granulite is composed of olivine + orthopyroxene. This mineral association can be generated at $670^{\circ} \mathrm{C}$ independently of the pressure conditions (Bucher and Frey, 1994) and is stable in all temperatures from the

The aluminous granulites are comprised of garnet + plagioclase + sillimanite + biotite + quartz \pm potassic feldspar \pm cordierite. According to White et al. (2007), this association indicates temperature conditions exceeding $720^{\circ} \mathrm{C}$ and pressure up to $8.5 \mathrm{kbar}$. The geothermobarometric data obtained by means of conventional geothermometers and geobarometers resulted in relatively low temperatures of $630-716{ }^{\circ} \mathrm{C}$ and pressures ranging from 4.9 to $8.3 \mathrm{kbar}$. process which accompanied the exhumation of the Acaiaca Complex that was followed by partial mylonitization and gneissification of the granulites. The most likely metamorphic conditions for the formation of mafic granulites were $768 \pm 28^{\circ} \mathrm{C}$ and $9.9 \pm 1.6 \mathrm{kbar}$. The mineral assemblage of granulite facies (orthopyroxene + clinopyroxene + plagioclase + hornblende) can be the result of reaction (1), which depicts the consumption of hornblende (Spear, 1995). As this is a divariant equilibrium reaction, reactants and products can coexist as an assemblage within a range of temperatures. granulites, but are not in equilibrium with the main mineral association. Blue-green hornblende was generated at the expense of brownish hornblende and pyroxene. Cummingtonite replaces orthopyroxene (reaction 2, Spear, 1995).

Orthopyroxene + quartz $+\mathrm{H}_{2} \mathrm{O}=$ cummingtonite

coronas can be produced by reaction (3), while the garnet-ilmenite symplectites can be the product of reaction (4). According to Harley (1989), reaction (3) may be indicative of a retrometamorphic trajectory characterized by near-isobaric cooling.

Orthopyroxene + plagioclase + quartz $=$ garnet

$$
\mathrm{FeO}_{\text {ilmenite }}+\text { plagioclase }=\text { garnet }
$$

middle amphibolite to the granulite facies. Anthophyllite, chlorite, serpentine and talc occur as replacement products of olivine and pyroxene. The appearance of anthophyllite, talc and serpentine may be due to reactions (5), (6) and (7) by con- sumption of orthopyroxene \pm olivine by the introduction of an aqueous fluid phase. Reaction (5) indicates intake of silica, possibly derived from siliceous country rocks and introduced by the circulating aqueous fluids.

$$
\begin{gathered}
\text { Orthopyroxene }+ \text { quartz }+\mathrm{H}_{2} \mathrm{O}=\text { anthophyllite } \\
\text { Orthopyroxene }+\mathrm{H}_{2} \mathrm{O}=\text { serpentine }+ \text { talc } \\
\text { Olivine + orthopyroxene }+\mathrm{H}_{2} \mathrm{O}=\text { serpentine }
\end{gathered}
$$

Those obtained by THERMOCALC provided temperatures and pressures of $712 \pm 79^{\circ} \mathrm{C}$ and $5.0 \pm 0.7 \mathrm{kbar}$, consistent with the granulite facies metamorphism. The generation of the mineral association of this lithotype can be associated with the crystallization of melts generated by anatexis. According to the literature (e.g. Vernon and Johnson, 2000; Johnson et al., 2001; Marchildon and Brown, 2003; Vernon et al., 2003; Vernon and Clarke, 2008) the occurrence of well-formed cordierite and plagioclase as found in this rock type (Figure $3 \mathrm{~d}$ ) is indicative of this process. Reaction (8) is a discontinuous reaction in the KFMASH-system at temperatures around $750^{\circ} \mathrm{C}$ and with melt generation at the costs of biotite dehydration in the absence of fluid (Spear et al., 1999). Reaction (9) in the CNKFMASH system is a variation of reaction (8) that considers the generation of melt in the presence of plagioclase (Johnson et al., 2001).

$$
\text { Biotite }+ \text { quartz }+\mathrm{Al}_{2} \mathrm{SiO}_{5}=\text { garnet }+ \text { cordierite }+ \text { potassic feldspar }+ \text { liquid }
$$

$$
\text { Biotite }+ \text { plagioclase } 1+\mathrm{Al}_{2} \mathrm{SiO}_{5}+\text { quartz }=\text { garnet }+ \text { cordierite }+ \text { plagioclase } 2+\text { potassic feldspar }+ \text { liquid }
$$


The garnet-kyanite-cordierite schist consists of a mineral association that is not in equilibrium, as indicated by the symplectitic intergrowths of cordierite with quartz and the replacement of staurolite, garnet, and kyanite by cordierite. The oldest mineral assemblage is typical of the amphibolite facies and consists of biotite + kyanite + staurolite + garnet + plagioclase + quartz . A younger generation of cordierite is partially replacing the previous minerals. According to Jordt-Evangelista (1984,

\section{Conclusions}

The Acaiaca Complex records a metamorphic event of granulite facies characterized by temperatures near to $800^{\circ} \mathrm{C}$ and pressure conditions between 5.0 and $9.9 \mathrm{kbar}$ that generated felsic, mafic, ultramafic and aluminous granulites. Both the felsic and the aluminous granulites were submitted to anatectic processes during the granulite facies metamorphism. The partial melting of felsic granulites resulted in pegmatites of granitic composition (Figure 2). Anatexis of aluminous granulites gave

\section{Acknowledgements}

E. Medeiros Júnior thanks CAPES for the doctoral scholarship. G. Queiroga gratefully acknowledges grants

\section{References}

1985) the appearance of cordierite in these rocks marks the transition of the amphibolite facies to the granulite facies. According to Holdaway and Lee (1977) and Bucher and Frey (1994), rocks rich in minerals such as garnet, cordierite, calcic plagioclase and free of potassic feldspar may represent an anatectic restite. The garnet-kyanite-cordierite granulite is more magnesian $(8.9$ to $11.5 \mathrm{wt} \%$ of $\mathrm{MgO})$ than the garnet-sillimanite granulite (0.8 to $3.1 \mathrm{wt} \% \mathrm{MgO}$ ) (Medeiros Junior and Jordt-Evangelista, 2010). The compositional and mineralogical difference between the two types of aluminous granulites, especially regarding the $\mathrm{MgO}$ content, support the interpretation that an anatectic process occurred during the granulite facies metamorphism of the aluminous granulites. The Mg-rich garnet- kyanite-cordierite schist could be the restite and the $\mathrm{Mg}$-poor garnetsillimanite granulite could be the product of the crystallization of the melt as also indicated by the well-formed cordierite and plagioclase crystals. rise to garnet-kyanite-cordierite schist as restite and sillimanite-garnet granulite as the product of the melt crystallization. Minerals such as staurolite, belonging to the oldest association of lower metamorphic grade, are occasionally preserved during the progressive metamorphism. The retrometamorphic path is characterized by a near-isobaric cooling. The gneissification of part of granulites is the last metamorphic record possibly related to the exhumation of the AC.

provided by the DAAD - German and CAPES - Brazil organizations for a research stay at TU Bergakademie,
The age of granulite facies metamorphic event was established by Th-U-Pb monazite geochronology. Both ortho and a para-derived dated granulite samples display an age of around $2060 \mathrm{Ma}$, similar to the age mentioned by Noce et al. (2007) for the Mantiqueira Complex metamorphism (2085 - $2041 \mathrm{Ma})$. Thus, it is possible to state that the Acaiaca Complex is a high grade metamorphic unit geochronologically related to the Mantiqueira Complex.

Freiberg. H. Jordt-Evangelista thanks FAPEMIG for financial support (Project APQ-00732-12).

BHATTACHARYA, A., MAZUMDAR, A.C., SEN, S.K. Fe-Mg mixing in cordierite: Constraints from natural data and implications for cordierite-garnet geothermometry in granulites. American Mineralogist, v. 73, p. 338-344, 1988.

BUCHER, K., FREY, M. Petrogenesis of Metamorphic Rocks. Berlim: Springer, 1994. 318p.

DE WARD, D. A proposed subdivision of the granulite facies. American Journal of Science, v. 263, n. 4, p. 55-461, 1965.

DONOVAN, J.J., HANCHAR, J.M., PICOLLI, P.M., SCHRIER, M.D., BOATNER, L.A., JAROSEWICH, E. A re-examination of the rare-earth element orthophosphate standards in use for electron-microprobe analysis. Canadian Mineralogist, v. 41, p. 221-232, 2003.

FETTES, D., DESMONS J. Metamorphic Rocks: A Classification and Glossary of Terms: Recommendations of the International Union of Geological Sciences Subcommission on the Systematics of Metamorphic Rocks. Cambridge University Press, 2007. 244p.

HARLEY, S.L. The origins of granulites: a metamorphic perspective. Geological Magazine, v. 126, n. 3, p. 215-247, 1989.

HOLDAWAY, M.J., LEE, S.M. Fe-Mg cordierite stability in high grade pelitic rocks based on experimental, theoretical and natural observations. Contributions to Mineralogy and Petrology, v. 63, n. 2, p. 175-198, 1977.

HOLLAND, T., BLUNDY, J. Non-ideal interaction in calcic amphiboles and their bearing on amphibole-plagioclase thermometry. Contributions to Mineralogy and Petrology, v. 116, n. 4, p. 433-447, 1994. 
JAROSEWICH, E., BOATNER, L.A. Rare-earth element reference samples for electron microprobe analysis. Geostandards Newsletter, v. 15, p. 397-399, 1991.

JOHNSON, T.E., HUDSON, N.F.C., DROOP, T.R. Partial melting in the Inzie Head gneisses: the role of water and a petrogenetic grid in KFMASH applicable to anatectic pelitic migmatites. Journal of Metamorphic Geology, v. 19, p. 99-118, 2001.

JORDT-EVANGELISTA, H. Petrologische Untersuchungen im Gebiete zwischen Mariana und Ponte Nova, Minas Gerais, Brasilien. Alemanha: Universidade Técnica de Clausthal, 1984. 183f (PhD thesis).

JORDT-EVANGELISTA, H. Petrologia de fases, geotermometria e geobarometria do Complexo Granulítico de Acaiaca, Sudeste do Quadrilátero Ferrífero, MG. In: SIMP. GEOL. MINAS GERAIS, 3, 1985. Belo Horizonte, Anais... (Annals...) Belo Horizonte: SBG, 1985, 165-178.

JORDT-EVANGELISTA, H., MULLER, G. Petrology of a transition zone between the Archean Craton and the Coast Belt, SE of the Iron Quadrangle, Brazil. Chemie der Erde, v. 45, p.129-145, 1986a.

JORDT-EVANGELISTA, H., MULLER, G. Petrologia da Zona de Transição entre o Cráton do São Francisco e o Cinturão Móvel Costeiro na Região Sudeste do Quadrilátero Ferrífero, MG. In: CONGR. BRAS. GEOL., 34, 1986b. Goiânia, Anais... (Annals...) Goiânia: SBG, 1986, p. 1471-1479.

KOZIOL, A.M. Recalibration of the garnet-plagioclase-Al2SiO5-quartz (GASP) geobarometer and applications to natural paragenesis. EOS, v. 70, n. 15, p. 493, 1989.

KOZIOL, A.M., NEWTON, R.C. Redetermination of the anorthite breakdown reaction and improvement of the plagioclase-garnet- $\mathrm{Al}_{2} \mathrm{SiO}_{5}$-quartz geobarometer. American Mineralogist, v. 73, p. 216-223, 1988.

KRETZ, R. Transfer and exchange equilibria in a portion of the pyroxene quadrilateral as deduced from natural and experimental data. Geochimica et Cosmochimica Acta, v. 46, p. 411-422, 1982.

LEAKE, B.E., WOOLLEY, A.R., ARPS, C.E.S., BIRCH, W.D., GILBERT, M.C., GRICE, J.D., HAWTHORNE, F.C., KATO, A., KISCH, H.J., KRIVOVICHEV, V.G., LINTHOUT, K., LAIRD, J., MANDARINO, J.A., MARESCH, W.V., NICKEL, E.H., ROCK, N.M.S., SCHUMACHER, J.C., SMITH, D.C., STEPHENSON, N.C.N., UNGARETTI, L., WHITTAKER, E.J.W., YOUZHI, G. Nomenclature of amphiboles: report of the Subcommittee on Amphiboles of the International Mineralogical Association Commission on New Minerals and Mineral Names. Eur. J. Mineral., v. 9, p. 623-651, 1997.

LUDWIG, K.R. Users manual for Isoplot/Ex rev. 2.49. A geochronological toolkit for Microsoft Excel. Berkeley Geochronology Center Special Publication, v. 1a, p. $1-55,2001$.

MARCHILDON, N., BROWN, M. Spatial distribution of melt-bearing structures in anatectic rocks from Southern Brittany, France: implications for melt transfer at grain- to orogen-scale. Tectonophisics, v. 364, p. 215-235, 2003.

MEDEIROS JUNIOR, E.B. Petrogênese do Complexo Acaiaca, MG. Ouro Preto: Universidade Federal de Ouro Preto, 2009. 101f (Master's thesis).

MEDEIROS JÚNIOR, E.B., JORDT-EVANGELISTA, H. Petrografia e geoquímica dos granulitos do Complexo Acaiaca, região centro-sudeste de Minas Gerais. REM- Revista Escola de Minas, v. 63, n. 2, p. 219-228, 2010.

MONTEL. J.M., FOREST, S., VESCHAMBRE, M., NICOLLET, C., PROVOST, A. A fast, reliable, inexpensive in-situ dating technique: Electron microprobe ages on monazite. Chemical Geology, v. 131,p. 37-53, 1996.

NEWTON, R.C. \& HASELTON, H.T. Thermodynamics of the garnet-plagioclase$-\mathrm{Al}_{2} \mathrm{SiO}_{5}$-quartz geobarometer. In: NEWTON, R.C., NAVROTSKY, A., WOOD, B.J. (Ed.) Thermodynamics of Minerals and Melts. New York: Springer-Verlag, 1981. cap. 7, p. 131-147. 293 p.

NOCE, C.M, PEDROSA-SOARES, A.C., SILVA, L.C., ARMSTRONG, R., PIUZANA D. Evolution of polycyclic basement complexes in the Araçuaí Orogen, based on U-Pb SHRIMP data: Implications for Brazil-Africa links in Paleoproterozoic time. Precambrian Research, v. 159, p. 60-78, 2007.

POWELL, R., HOLLAND, T. Optimal geothermometry and geobarometry. American Mineralogist, v. 79, p. 120-133, 1994.

SCHULZ, B., BRÄTZ, H., BOMBACH, K., KRENN, E. In-situ Th-Pb dating of monazite by $266 \mathrm{~nm}$ laser ablation and ICP-MS with a single collector, and its control 
by EMP analysis. Zeitsch. Angewandte Geologie, v. 35, p. 377-392, 2007.

SPEAR, F.S. Metamorphic Phase Equilibria and Pressure-Temperature-Time Paths.

Washington: Mineralogical Society of America Monograph, 1995. 799p.

SPEAR, F.S., KOHN, M.J., CHENEY, J.T. P-T paths from anatectic pelites. Contributions to Mineralogy and Petrology, v. 134, p. 17-32, 1999.

SUZUKI, K., ADACHI, M., KAJIZUKA, I. Electron microprobe observations of Pb diffusion in metamorphosed detrital monazites. Earth and Planetary Science Letters, v. 128, p. 391-405, 1994.

TEIXEIRA, W., JORDT-EVANGELISTA, H., KAWASHITA, K. \& TAYLOR P.N. 1987. Complexo Granulítico de Acaiaca, MG: idade, petrogênese e implicações tectônicas. In: SIMP. GEOL. MINAS GERAIS, 4, 1987. Belo Horizonte, Anais... (Annals...) Belo Horizonte: SBG, 1987. p. 58-71.

THOMPSON, A.B. Mineral reactions in pelitic rocks: II. Calculation of some P-T-x (Fe-Mg) phase relations. American Journal of Sciences, v. 276, p. 425-454, 1976.

VERNON, R.H., CLARKE, G.L. Principles of Metamorphic Petrology. New York: Cambridge University Press, 2008. 446p.

VERNON, R.H., COLLINS, W.J. \& RICHARDS, S.W. Contrasting magmas in metapelitic and metapsammitic migmatites in the Cooma Complex, Australia. Visual Geosciences, v. 8, p. 45-54, 2003.

VERNON, R.H, JOHNSON, S.E. 2000. Transition from gneiss to migmatite and the relationship of leucosome to peraluminous granite in the Cooma Complex, SE Australia. In: JESSELL, M.W., URAI, J.L. (Ed.) Stress, strain and structure. A volume in honour of W.D. Means. Journal of the Virtual Explorer, v. 2 (print \& CD). 2000

WELLS, P.R.A. Chemical and thermal evolution of Archaen sialic crust, southern West Greenland. Journal of Petrology, v. 20, n. 2, p. 187-226, 1979.

WHITE, R.W., POWELL, R., HOLLAND, T.J.B. Progress relating to calculation of partial melting equilibria for metapelites. Journal of Metamorphic Geology, v. 25, p. 511-527, 2007.

Received: 8 September 2015 - Accepted: 2 December 2015.

Erratum

In the Geosciences Article "Electron microprobe Th-U-Pb monazite dating and metamorphic evolution of the Acaiaca Granulite Complex, Minas Gerais, Brazil", published in the Revista Escola de Minas 2016; 69(1): 21-32.

\section{Which reads:}

Edgar Batista Medeiros Júnior

Professor Assistente, Universidade Federal do Espírito Santo - UFES, Departamento de Geologia Alegre - Espírito Santo - Brasil - edgarjr@ymail.com Hanna Jordt-Evangelista

Professora Titular, Universidade Federal de Ouro Preto - UFOP, Escola de Minas, Departamento de Geologia Ouro Preto - Minas Gerais - Brasil hanna@degeo.ufop.br

Gláucia Nascimento Queiroga

Professora Adjunta, Universidade Federal de Ouro Preto - UFOP, Escola de Minas, Departamento de Geologia Ouro Preto - Minas Gerais - Brasil glauciaqueiroga@yahoo.com.br

Bernhard Schulz

Professor, TU Bergakademie - Institute of Mineralogy Freiberg - Saxony - Germany - bernhard.schulz@mineral.tu-freiberg.de Rodson Abreu Marques

Professor Adjunto, Universidade Federal do Espírito Santo - UFES, Departamento de Geologia Alegre - Espírito Santo - Brasil - rodson.marques@ufes.br

\section{Should be read as:}

Edgar Batista Medeiros Júnior

Professor Assistente, Universidade Federal do Espírito Santo - UFES, Departamento de Geologia Alegre - Espírito Santo - Brasil - edgarjr@ymail.com Reik Degler

Doutorando da Universidade Federal de Minas Gerais - Departamento de Geociências - Programa de Pós-Graduação em Geologia - Belo Horizonte - Minas Gerais Brasil - reikdegler@gmail.com

Hanna Jordt-Evangelista

Professora Titular, Universidade Federal de Ouro Preto - UFOP, Escola de Minas, Departamento de Geologia Ouro Preto - Minas Gerais - Brasil -

hanna@degeo.ufop.br

Gláucia Nascimento Queiroga

Professora Adjunta, Universidade Federal de Ouro Preto - UFOP, Escola de Minas, Departamento de Geologia Ouro Preto - Minas Gerais - Brasil -

glauciaqueiroga@yahoo.com.br

Bernhard Schulz

Professor, TU Bergakademie - Institute of Mineralogy Freiberg - Saxony - Germany - bernhard.schulz@mineral.tu-freiberg.de

Rodson Abreu Marques

Professor Adjunto, Universidade Federal do Espírito Santo - UFES, Departamento de Geologia Alegre - Espírito Santo - Brasil - rodson.marques@ufes.br 